\title{
Demo: VibKeyboard: Virtual Keyboard Leveraging Physical Vibration
}

\author{
Jian Liư ${ }^{\dagger}$ Yingying Chen ${ }^{\dagger}$, Marco Gruteser $\$$ \\ †Stevens Institute of Technology, Hoboken, NJ 07030, USA \\ ${ }^{\S}$ Rutgers University, North Brunswick, NJ 08902, USA \\ †\{jliu28, yingying.chen\}@stevens.edu, `gruteser@winlab.rutgers.edu
}

\begin{abstract}
VibKeyboard could accurately determine the location of a keystroke on extended surface areas leveraging a single vibration sensor. Unlike capacitive sensing, it does not require conductive materials and compared to audio sensing it is more robust to acoustic noise. In VibKeyboard, the received vibration signals are determined by the location of the touch impact. This allows location discrimination of touches precise enough to enable emerging applications such as virtual keyboards on ubiquitous surfaces for mobile devices. VibKeyboard seeks to extract unique features in frequency domain embedded in the vibration signal attenuation and interference and perform fine grained localization. Our experimental results demonstrate that VibKeyboard could accurately recognize keystrokes from close-by keys on a nearby virtual keyboard.
\end{abstract}

\section{INTRODUCTION}

As the form factor of our mobile and wearable devices shrinks, there exists an increasing need to support interaction beyond the confines of the device itself. Particularly small touchscreens and interfaces can render complex input cumbersome. In order to tackle this constraint, we present VibKeyboard which uses physical vibration to enable typing on any surface such as a table to interact with mobile devices.

Vibration effects caused by diverse signal attenuation and interference have high potential to discriminate the propagation conditions in a fine-grained manner. Such effects can be utilized to support a broad array of application domains such as virtual keyboards, which are currently limited by complex hardware requirements and environmental noises.

Existing approaches either use acoustic signals [6,7] or laser projections [4] to construct virtual keyboards. Compared to acousticbased approaches, vibration-based sensing is more robust to various environmental sounds. And the approach only requires a single low-cost vibration receiver, making it scalable comparing to the laser projection based solution.

When users touch a surface, each touch generates vibration signals with different frequencies. The vibration signals spread out in the medium and experience various attenuation and interference re-

Permission to make digital or hard copies of part or all of this work for personal or classroom use is granted without fee provided that copies are not made or distributed for profit or commercial advantage and that copies bear this notice and the full citation on the first page. Copyrights for third-party components of this work must be honored. For all other uses, contact the owner/author(s).

MobiCom'16 October 03-07, 2016, New York City, NY, USA

(C) 2016 Copyright held by the owner/author(s).

ACM ISBN 978-1-4503-4226-1/16/10.

DOI: http://dx.doi.org/10.1145/2973750.2985624

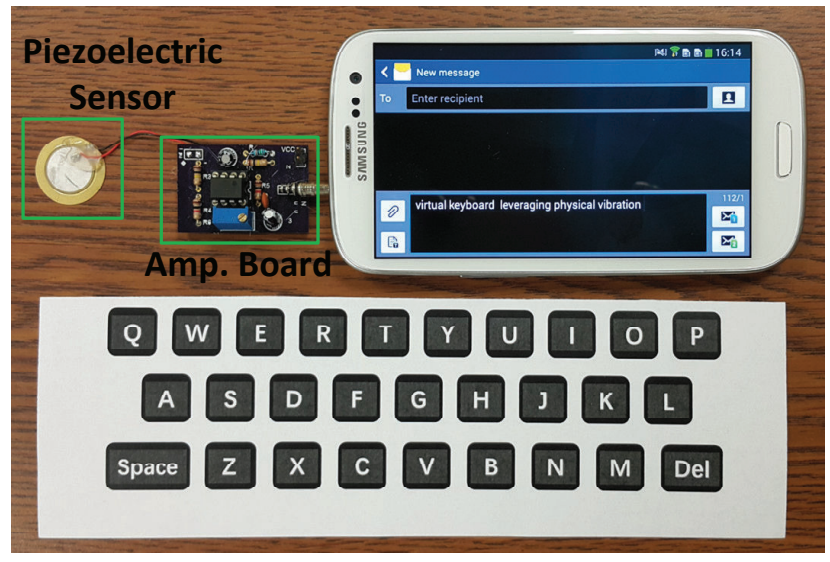

Figure 1: The VibKeyboard platform prototype.

sulted from different surface materials and multiple paths dominated by the location of the touch on the surface. The diverse vibration signals could be exploited to discriminate the fine-grained location of each touch on the surface via fingerprint-based approaches. In this work, we build an ubiquitous keyboard leveraging a low-cost vibration sensor for mobile devices as shown in Figure 1.

\section{SYSTEM DESIGN}

Particularly, VibKeyboard takes as input time-series amplitude measurements of vibration signals from a vibration receiver. After receiving the vibration signals, the system performs Vibration Detection \& Segmentation to detect and obtain the useful segment of the received vibration signals. Next, the system utilizes the Vibration Feature Extraction to extract the unique vibration feature (e.g., power spectrum density) from the segmented signals in the frequency domain.

Next, the extracted vibration features are used by two phases in VibKeyboard: profiling and identification. In the profiling phase, the extracted features are labeled with corresponding ground truth, and saved to build an object profile. In the identification phase, the collected vibration samples are used to extract vibration features, which serve as inputs to a vibration classifier via SVM. The classifier compares the extracted features with the signatures in the preconstructed profile to identify the target object and determine its location.

Vibration Detection \& Segmentation. After receiving vibration signals, VibKeyboard utilizes an energy-based approach to detect and determine the segment of useful vibration signals. In particular, it calculates the short time energy levels of the received vibration 
Table 1: Comparison of vibration receiving sensors: geophone, accelerometer and piezoelectric sensors.

\begin{tabular}{|c|c|c|c|}
\hline & Sampling rate & Max. vibration freq. & Cost \\
\hline Geophone [2] & (Analog output) & $0.4 \mathrm{kHz}$ & $\sim \$ 50.00$ \\
\hline Accel. [1] & $0.1 \mathrm{~Hz} \sim 3.2 \mathrm{kHz}$ & $1.6 \mathrm{kHz}$ & $\sim \$ 4.00$ \\
\hline Piezoelectric & (Analog output) & $\geqslant \mathbf{2 0 ~ k H z}$ & $\sim \$ \mathbf{1 . 0 0}$ \\
\hline
\end{tabular}

signals by accumulating the square of their amplitudes in a sliding time window. We then use a threshold-based approach to detect the segment of useful vibration signals.

Vibration Feature Extraction. VibKeyboard utilizes the power spectral density (PSD) of the received vibration signals as the basis for feature extraction to perform localizing keystrokes. The PSD reflects the power distribution of the sensed vibration signals at each specific frequency, which can well capture the attenuation and interference effects influenced by vibration source, propagation medium, and objects contacting the medium surface. The PSD of a received vibration signal $r_{i}$ can be estimated by:

$$
P S D_{i}=10 \log _{10} \frac{\left(a b s\left(F F T\left(r_{i}\right)\right)\right)^{2}}{f_{s} \times n},
$$

where $n$ is the number of samples of the received signal $r_{i}, f_{s}$ is the sampling rate, and $F F T(\cdot)$ is the fast Fourier transform operation.

Vibration Classification. During the profiling phase, VibKeyboard constructs a set of keystrokes profiles with the vibration features (i.e., PSD) by labeling vibration signals collected from different keystrokes. In the later identification phase, when there is a vibration signal detected and segmented, VibKeyboard needs to extract the vibration feature from the segmented signal and classify the feature by matching it to the existing keystroke profiles. Specifically, a vibration classifier is built based on the Support Vector Machine (SVM) using LIBSVM [5] and the linear kernel function.

\section{HARDWARE PLATFORM IMPLEMEN- TATION}

In order to achieve fine-grained localization using physical vibration, our system needs to be capable of sensing the vibration signals in a wide frequency range to fully cover the frequency information of the vibration signals caused by keystrokes. Additionally, we need to design a platform that can be easily connected to mobile devices to facilitate mobile vibration-based sensing applications such as virtual keyboard.

Toward this goal, we design and implement a low-cost vibration receiver as shown in Figure 1, which consists of a vibration sensor and a low-power consumption amplifier. It can be easily connected to mobile devices via $3.5 \mathrm{~mm}$ audio jack. We devise a low-cost passive vibration sensor, piezoelectric sensor, to collect vibration signals. Compared to other types of popular commercial vibration sensors shown in Table 1: Geophone sensors and capacitive MEMS accelerometers, the piezoelectric sensor has the largest frequency response range and the lowest cost. Moreover, the sensor is so small (i.e., 0.48 square inches in area) that can be easily attached to any solid surface and integrated with a smartphone for sensing vibration signals.

With a mobile device, we could use its internal audio component (i.e., microphone ADC) to convert the analog signals obtained from the piezoelectric sensor to the digital signals. Additionally, we use an operational amplifier $T L C 272$ [3] to increase both positive and negative voltage analog signals with a single power supply. Upon the available sampling rates in the microphone ADC of most commercial mobile devices, the designed mobile hardware platfor$\mathrm{m}$ samples the piezoelectric sensor readings at $48 \mathrm{kHz}$.

\section{DEMONSTRATION SETUP}

We need a table (a normal office table is enough) to hold several mobile phones with developed sensor boards, and our piezoelectric sensors need to be attached on the table by using double-sided tapes. A virtual keyboard printed on a paper is also needed to be placed on the table to guide users' typing.

\section{ACKNOWLEDGEMENT}

This work was partially supported by the National Science Foundation Grants CNS1409767, CNS1514436 and SES1450091.

\section{REFERENCES}

[1] Ad accelerometer adxl345. http://www.analog.com/media/en/technicaldocumentation/data-sheets/ADXL345.pdf.

[2] Oyo geospace gs-11d. http://www.geospace.com/geophones-gs-11d/.

[3] Ti operational amplifier tlc272. http://www.ti.com/product/tlc272.

[4] Projection keyboard vk200. http://www.ctxtechnologies.com /products/vk-200-keyfob-virtual-keyboard/, 2015.

[5] C.-C. Chang and C.-J. Lin. Libsvm: A library for support vector machines. ACM Transactions on Intelligent Systems and Technology (TIST), 2(3):27, 2011.

[6] J. Wang, K. Zhao, X. Zhang, and C. Peng. Ubiquitous keyboard for small mobile devices: harnessing multipath fading for fine-grained keystroke localization. In $A C M$ MobiSys, pages 14-27, 2014.

[7] R. Xiao, G. Lew, J. Marsanico, D. Hariharan, S. Hudson, and C. Harrison. Toffee: enabling ad hoc, around-device interaction with acoustic time-of-arrival correlation. In $A C M$ MobileHCI, pages 67-76, 2014. 